\title{
Apatinib inhibits cellular invasion and migration by fusion kinase KIF5B-RET via suppressing RET/Src signaling pathway
}

\author{
Chen Lin ${ }^{1, *}$, Shanshan Wang ${ }^{1, *}$, Weiwei Xie ${ }^{1}$, Rongliang Zheng ${ }^{2}$, Yu Gan ${ }^{3}$, Jianhua \\ Chang $^{1}$ \\ ${ }^{1}$ Department of Medical Oncology, Fudan University Shanghai Cancer Center, Shanghai, 200032, P.R. China \\ ${ }^{2}$ Department of Nuclear Medicine, Sun Yat-Sen University Cancer Center, Guangzhou, 510000, P.R. China \\ ${ }^{3}$ State Key Laboratory of Oncogenes and Related Genes, Shanghai Cancer Institute, Renji Hospital, Shanghai Jiao Tong \\ University School of Medicine, Shanghai, 200032, P.R. China \\ "These authors contributed equally to this work
}

Correspondence to: Jianhua Chang, email: changjianhua@163.com

Yu Gan, email: ganyu@shSCI.org

Keywords: apatinib, KIF5B-RET, invasion and migration, Src signaling pathway, targeted therapy

Received: April 23, 2016

Accepted: July 10, 2016

Published: August 01, 2016

\section{ABSTRACT}

The Rearranged during transfection (RET) fusion gene is a newly identified oncogenic mutation in non-small cell lung cancer (NSCLC). The aim of this study is to explore the biological functions of the gene in tumorigenesis and metastasis in RET gene fusion-driven preclinical models. We also investigate the anti-tumor activity of Apatinib, a potent inhibitor of VEGFR-2, PDGFR- $\beta$, c-Src and RET, in RET-rearranged lung adenocarcinoma, together with the mechanisms underlying. Our results suggested that KIF5B-RET fusion gene promoted cell invasion and migration, which were probably mediated through Src signaling pathway. Apatinib exerted its anti-cancer effect not only via cytotoxicity, but also via inhibition of migration and invasion by suppressing RET/Src signaling pathway, supporting a potential role for Apatinib in the treatment of KIF5B-RET driven tumors.

\section{INTRODUCTION}

Treatment strategies for non-small cell lung cancer (NSCLC) have evolved to an emphasis on molecularly targeted therapy based on genomic classification of patients [1]. The discovery of EGFR mutations and ALK rearrangements has remarkably revolutionized the therapeutic landscape of NSCLC. Traditional platinumbased chemotherapy is gradually replaced by targeted drugs, like EGFR tyrosine kinase inhibitors (erlotinib, geftinib, afatinib) and ALK inhibitors (crizotinib and ceritinib) even in the front-line setting [2-3]. To date, a series of molecular alterations, such as BRAF, PIK3CA, BRAF, HER2 mutations and ROS1 translocations, have been successively recognized as driver mutations in NSCLC [4]. The patient benefits from antagonizing genetic alterations have been demonstrated in excessive clinical trials, including prolonged survival and improved quality of life. Identification of oncogenic drivers has been highlighted in the era of precision medicine [5].

Between late 2011 and early 2012, KIF5B-RET fusion gene was firstly identified in NSCLC by four independent research groups [6-9]. It is formed by a pericentric inversion on chromosome 10, which constitutes a coiled coil domain and induces autophosphorylation of RET tyrosine kinase, thereby activates uncontrolled signal transduction and leads to tumorigenesis [10-11]. The fusion gene is mutually exclusive to other major mutations like EGFR, KRAS, ALK and BRAF and has been considered as a novel driver mutation in lung adenocarcinomas (LADC) [6-8]. The overall prevalence of RET fusion in lung adenocarcinomas is $1-2 \%$ according to existing literatures, and it increases to $6-19 \%$ for tumors without other genetic variants [12]. Although in vitro and xenograft model data support the tumorigenicity potential of RET chimeras, information about other biological functions, like proliferation, migration and invasion is lacking.

Apatinib, a novel, orally administered receptor tyrosine kinase (RTK) inhibitor, targets the vascular endothelial growth factor receptor-2 (VEGFR-2), RET, platelet-derived growth factor- $\beta$ (PDGFR- $\beta$ ), v-Src sarcoma viral oncogene homolog (c-Src), and stem cell factor receptor (c-Kit) [13-14]. Highly selective 
competition within the ATP-binding site of VEGFR-2 in the cell will block the downstream signal transduction and inhibit tumor angiogenesis [15]. Based on this mechanism, phase III clinical trials have been conducted aiming at gastric carcinoma in China and Apatinib has proved to be effective and safe in the treatment of advanced gastric cancer patients [16]. However, anti-tumor activity of Apatinib in RET-rearranged lung cancer has never been reported. Considering the huge population baseline of NSCLC patients worldwide, the treatment for RET fusionpositive NSCLC patients with RET inhibitors has great significance both in theory and in practice.

Herein, we explored the biological functions of the gene in tumorigenesis and metastasis in RET gene fusion-driven preclinical models. The anti-tumor activity of Apatinib was also evaluated to explore the therapeutic potential in RET fusion-driven LADC.

\section{RESULTS}

\section{Establishment of stable transfected cell lines}

KIF5B-RET gene in our research had two isomers, variant 2 and variant 4(mentioned next as KV2 and KV4). The RT-PCR method showed the mRNA over-expression of the fusion gene after transfection (Figure 1A). The stable transfected cell lines successfully expressed phosphorylated RET, suggesting that KIF5B-RET could automatically activate RET kinase (Figure 1B).

\section{KIF5B-RET fusion gene was capable of inducing malignant transformation}

In order to verify the malignant transformation ability of KIF5B-RET fusion gene, transformed and parental NIH3T3 cells $\left(5 \times 10^{6}\right)$ were injected subcutaneously to 6 -week-old female nude mice. Four weeks later, the tumors grew to a diameter of about $1 \mathrm{~cm}$ in $\mathrm{KV}-2$ and $\mathrm{KV}-4$ groups, and the parental NIH3T3 cells had no tumorigenicity (data not shown). We then collected the xenograft tumors and conducted HE staining. Morphologically, tissues were similar to sarcoma tissue, and more mitotic figures and abnormal nuclei could be seen (Figure 2), confirming that KIF5B-RET fusion gene could induce the malignant transformation of fibroblast cell lines of 3T3.

\section{The functional role of KIF5B-RET fusion gene in cell proliferation, migration and invasion}

We compared the cell proliferative and colonyforming abilities between KIF5B-RET transfected A549 and BEAS2B cells and the control groups, and found no significant difference in proliferation rate or colony number and size (Figure 3). The migration ability of KIF5B-RET transfected A549 cells and BEAS2B cells were detected by using transwell chambers. The results showed that more transfected cells intruded into bottom chamber than the negative control cells both in BEAS2B cells and A549 cells lines (Figure 4A, 4B). Wound-healing assay was used to evaluate the effect on A549 migration, as shown in Figure 4C, after 48 hours, A549-KV2 and A549-KV4 cells migrated significantly close to the scratched wound than negative control cells, displaying greater migration ability (Figure 4C). Tumor invasion assay, another characteristic contributing to cancer invasion and metastasis, was conducted in A549 cell line to assess invasiveness, and indicated that KIF5B-RET fusion gene could also promote invasion of A549 cells with KV2 or KV4 (Figure 4D).

\section{Signaling pathways involved in the migration and invasion of KIF5B-RET positive cells}

Since KIF5B-RET fusion gene could promote migration and invasion of A549 cells, we tried to explore the downstream signaling pathways. The phosphorylation levels of migration and invasion-related signaling molecules were tested and we found that $\mathrm{p}-\mathrm{Src}$ and p-ERK were remarkably up-regulated in KIF5BRET positive A549 cells (Figure 5A). We then used a series of concentration of MEK1 inhibitor PD98059, which targeted the upstream signaling pathway of ERK1/2, and Src inhibitor Dasatinib to treat the cells. The concentration was ensured as $16 \mu \mathrm{M}$ and $40 \mathrm{nM}$ to obviously inhibit the p-ERK and p-Src(Figure 5B, 5C), and at this certain concentration, Dasatinib was observed to significantly reduce migration and invasion of KIF5BRET positive A549 cells (Figure 5D, 5E), while PD98059 did not(Figure $5 \mathrm{~B}$ ), indicating that Src protein may be the downstream pathway of KIF5B-RET to mediate invasion and migration.

\section{Apatinib inhibited cell proliferation, migration and invasion of KIF5B-RET transfected A549 cells}

We evaluated Apatinib's capacity to inhibit cell growth in KIF5B-RET transfected A549 cells and the control groups, and observed a suppressed viability in a concentration-dependent manner although there was no obvious difference between the inhibition efficiency of KIF5B-RET transfected cells and the control groups (Figure 6A). We then analyzed the variation of protein expression by Western Blot, and found Apatinib caused a dose-independent reduction of the phosphorylation of RET gene fusion kinases and the p-RET was totally inhibited at the concentration of $8 \mu \mathrm{M}$, along with the inhibition of p-Src (Figure 6B). On this condition, the migration and invasion effects were significantly reduced in KIF5B-RET driven A549 cells, indicating Apatinib may be a promising anti-metastatic agent to reduce migration and invasion (Figure 6C, 6D). 
A

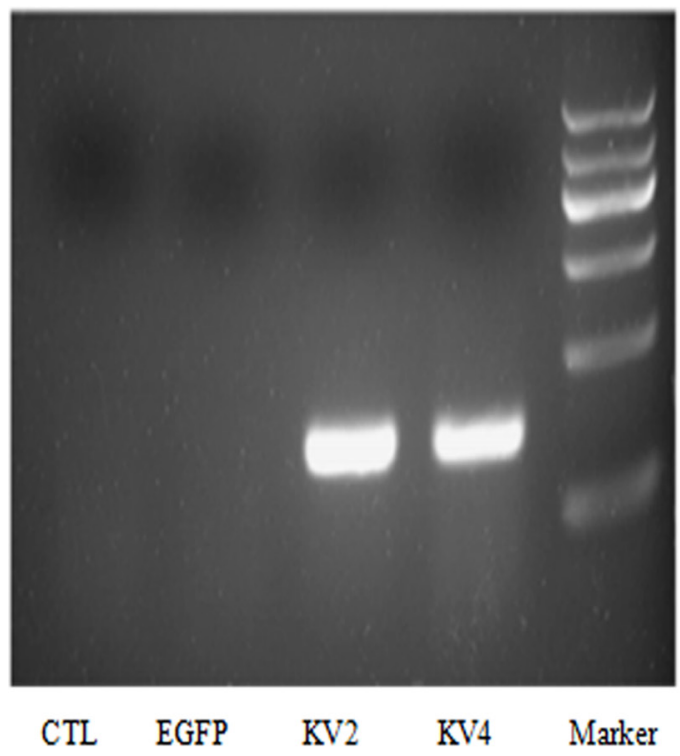

B

$\begin{array}{llll}\text { BEAS2B } & \text { BEAS2B } & \text { BEAS2B } & \text { BEAS2B } \\ - \text { KV2 } & - \text { KV4 } & \text {-EGFP } & - \text {-CTL }\end{array}$

$-1200$

$-900$

$-700$

$-500$

$-300$

$-100$

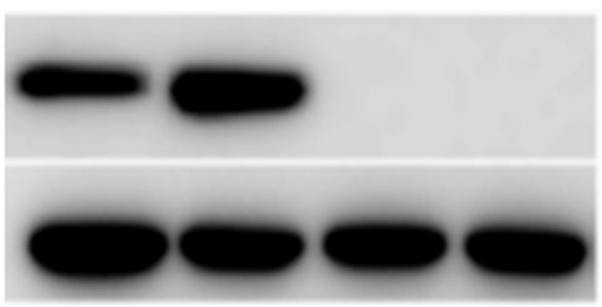

\section{A549-KV2 A549-KV4 A549-CTL}

p-RET

B-Actin

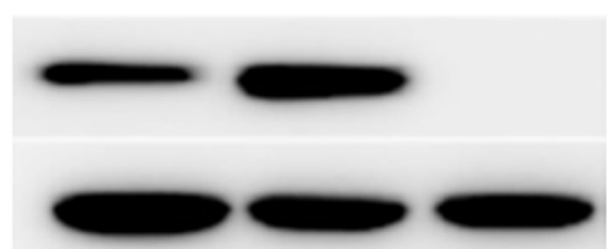

Figure 1: Establishment of stable transfected BEAS-2B and A549cell lines. (A) The RT-PCR method showed the mRNA overexpression of the fusion gene after transfection. (B) The stable transfected cell lines successfully expressed phosphorylated RET, suggesting that KIF5B-RET could automatically activate RET kinase.

A

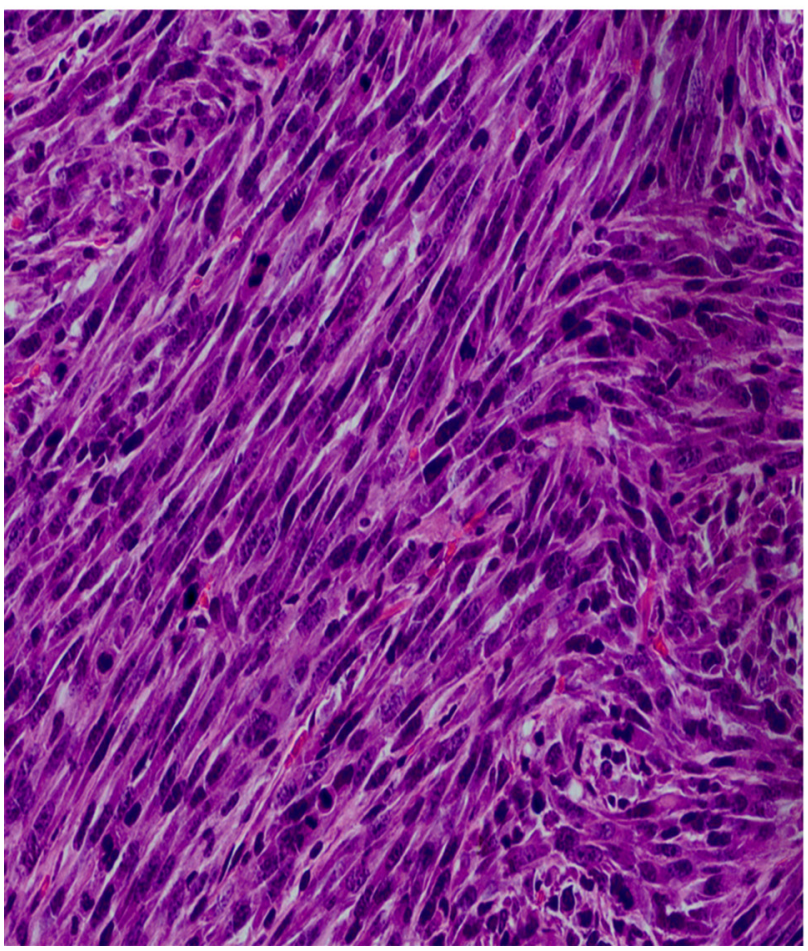

B

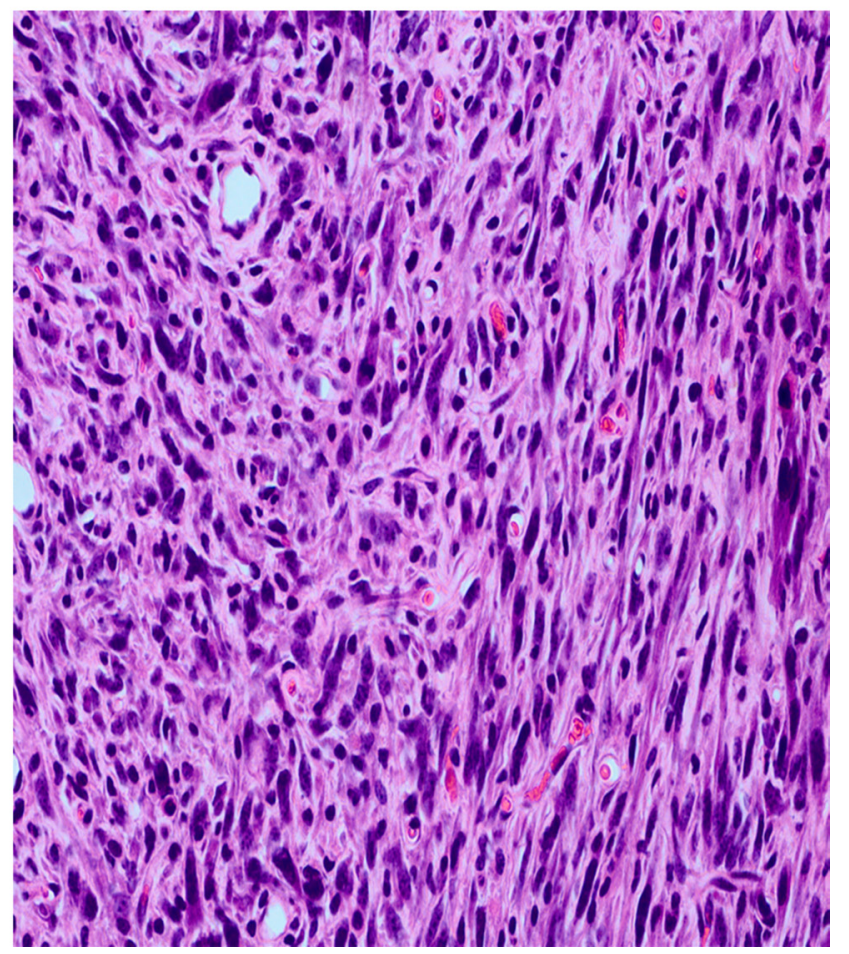

Figure 2: The HE staining of xenograft tumors of nude mice five weeks after subcutaneously injection of $5 \times 10^{6}$ 3T3cells carrying KIF5B-RET fusion gene. (A) Injection of 3T3 cells carrying KIF5B-RET-variant2 gene. (B) Injection of 3T3 cells carrying KIF5B-RET-variant4 gene. 
A

A549-CTL

A549-KV2

A549-KV4
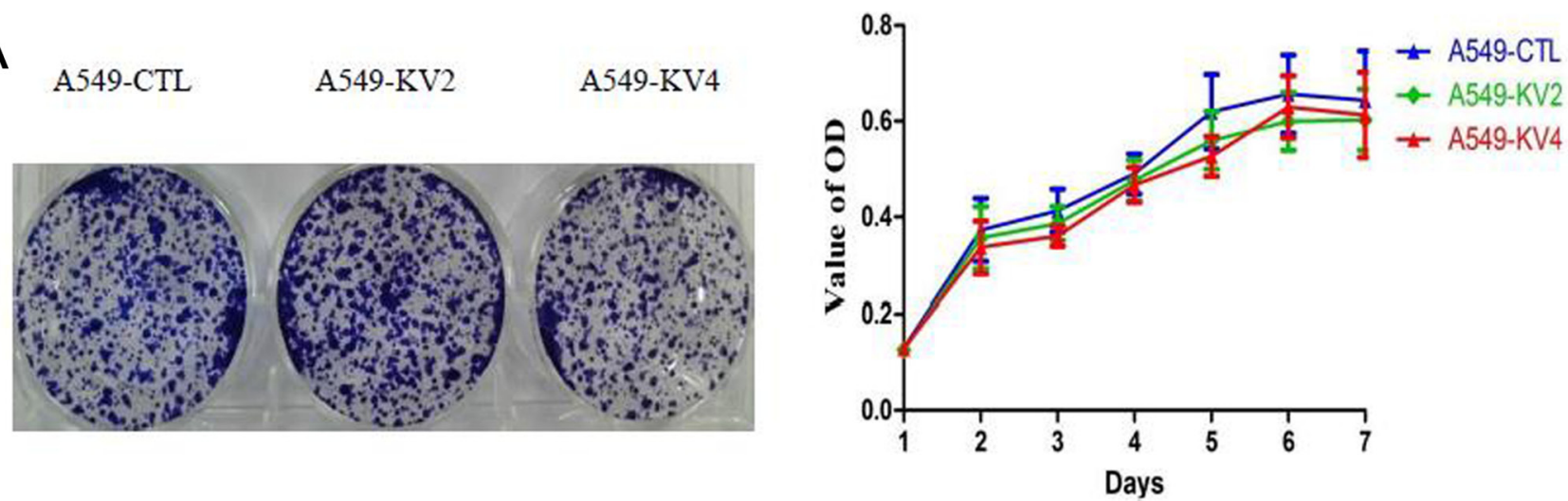

B

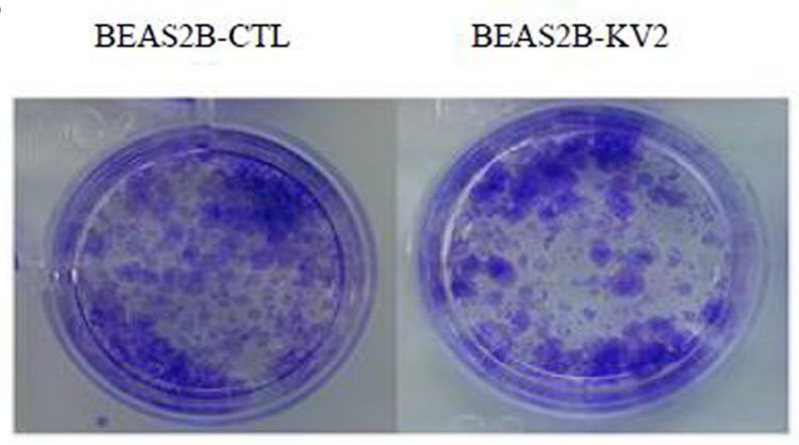

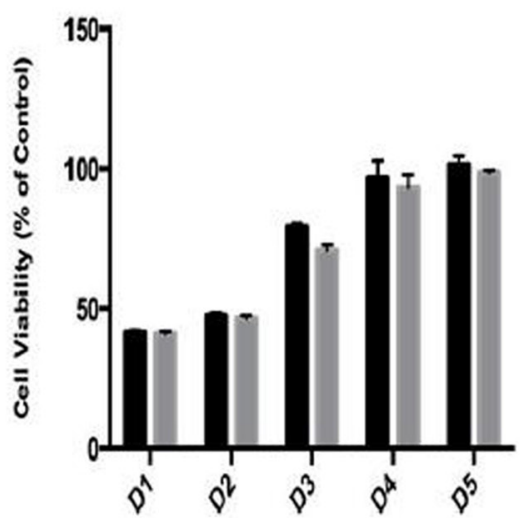

BEAS2B-CTL

BEAS2B-KV2

Figure 3: The proliferative and colony-forming abilities of KIF5B-RET transfected A549 and BEAS2B cells and the control groups. (A). A549 cells. (B). BEAS2B cells. The result showed no significant difference in proliferation rate or colony number and size.

A

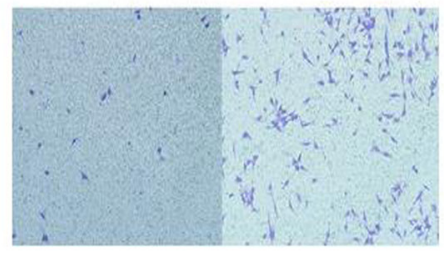

BEAS2B-CTL(10X) BEAS2B-KV2(10X)

C

Oh

$48 \mathrm{~h}$

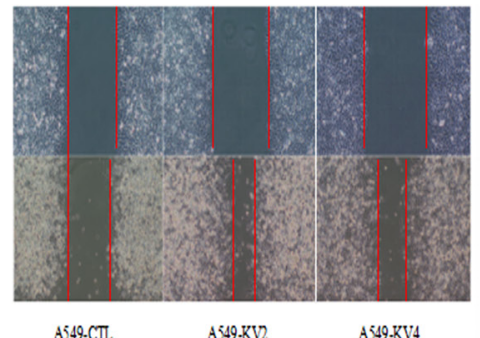

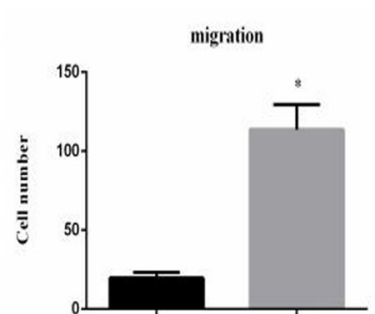

BEAS2B-CTL BEAS2B-KV2

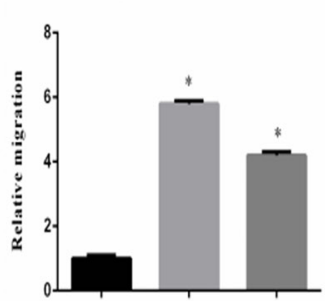

A59.CTL A59.KV2 A599.KVJ
B

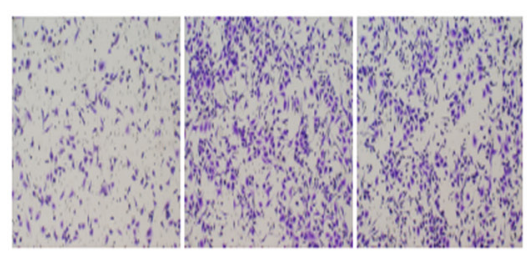

A549.CTL

A.49-KV2

A.49.KV4

D

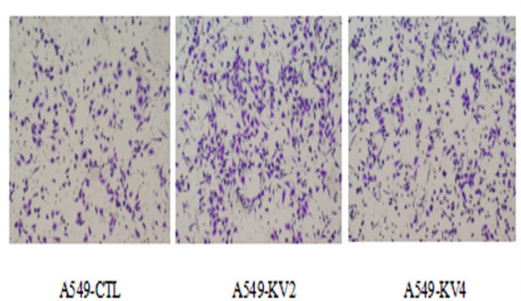

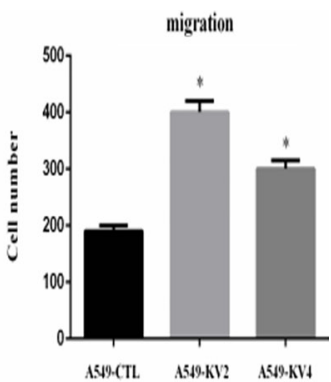

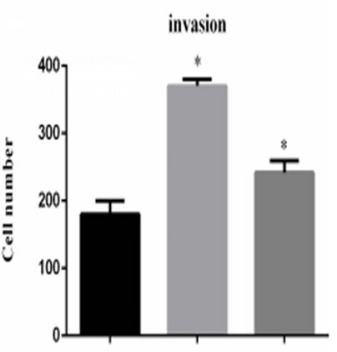

A599.CTL AS9-KV2 A599-KV/

Figure 4: KIF5B-RET fusion gene promoted migration and invasion of cancer cells in vitro. (A) Cell migration assay showed that KIF5B-RET promoted migration in BEAS2B-KV2 cells. (B) Cell migration assay showed that KIF5B-RET promoted migration in A549-KV2 and A549-KV4 cells. (C) Wound-healing assay showed that KIF5B-RET promoted migration in A549-KV2 and A549-KV4 cells. (D) Tumor invasion assay showed that KIF5B-RET promoted invasion in A549-KV2 and A549-KV4 cells. ${ }^{*} P<0.05$. 


\section{DISCUSSION}

In our study, we confirmed that KIF5B-RET expression in NIH3T3 cells could lead to oncogenic transformation, which was consistent with previous reports [7]. The functional characteristics of KIF5BRET were mainly focused on the proliferative effect in published literature, but whether KIF5B-RET affect the metastasis of human NSCLC cells has never been investigated. Our results showed that RET overexpression significantly enhanced migration and invasion of KIF5B-RET positive A549 cells and Src protein might be involved in the signaling pathways to mediate invasion and migration. This phenomenon was in a way consistent with the clinical implications that RET-rearranged tumors were prone to be more prevalent in patients at advanced stage or with metastatic diseases [17-19].This might facilitate the selection of patients for the screening of RET rearrangements and further treatment of RET inhibitors. However, we did not detect cell proliferation effect in KIF5B-RET transfected BEAS2B or A549 cells, which was different from the report of Qian et al., showing that KIF5B-RET fusion kinase promotes cell growth by multilevel activation of STAT3 in lung cancer [20].

Information about signal transduction downstream of the KIF5B-RET is still limited. Src, a membraneassociated nonreceptor tyrosine kinase, participates in the development of tumor metastasis through down-regulation of E-cadherin, up-regulation of matrix-degrading proteases (MMPs), activation of focal adhesion kinase (FAK), etc. [21-22]. In our experiment, compared with parental A549 cells, KIF5B-RET positive A549 cells had a stronger capability for migration and invasion, along with a remarkably up-regulation of p-Src. We then used Src inhibitor Dasatinib to treat the cells, and found migration and invasion ability of KIF5B-RET positive A549 cells was significantly reduced, indicating that Src protein is activated in the downstream pathway of KIF5B-RET driven tumors.
A

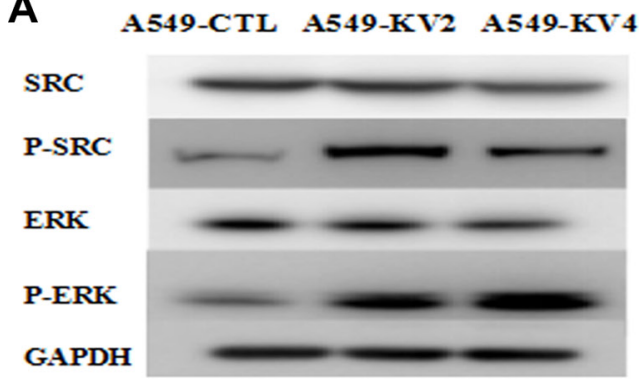

C

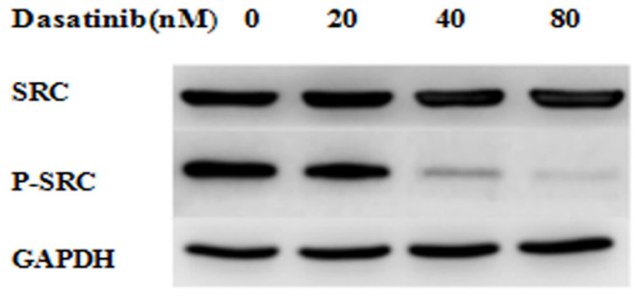

E

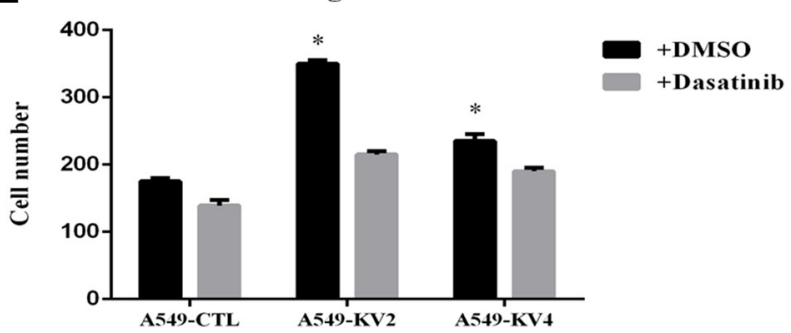

B

D

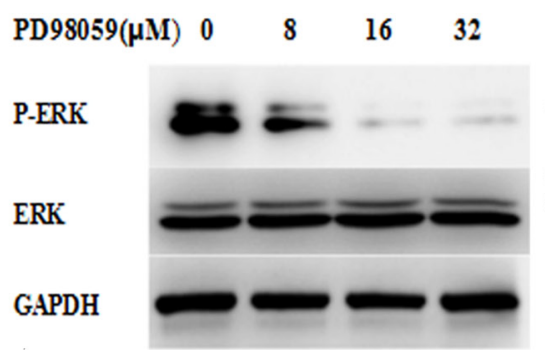

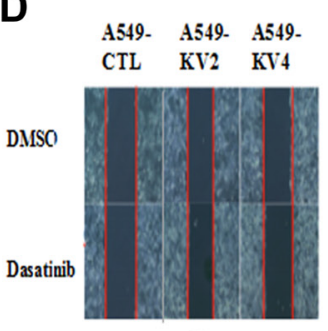

Oh
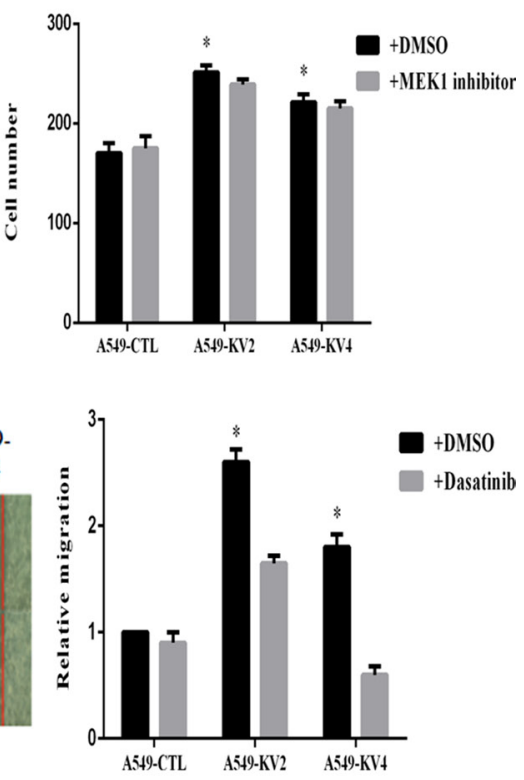

invasion

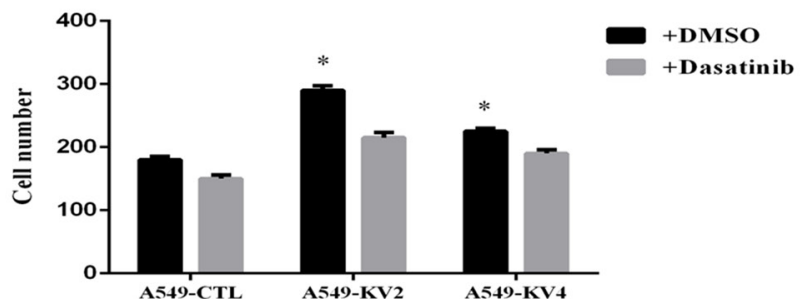

Figure 5: Signaling pathway involved in the migration and invasion of KIF5B-RET positive cells. (A) p-Src and p-ERK were remarkably up-regulated in KIF5B-RET positive A549 cells. (B) p-ERK was totally inhibited by PD98059 at the concentration of $16 \mu \mathrm{M}$. On this condition, no significant difference was shown in migration ability between KIF5B-RET positive A549 cells and negative control cells. (C) p-Src was obviously inhibited by Dasatinib at the concentration of $40 \mathrm{nM}$. (D) Treated with Dasatinib at a concentration of $40 \mathrm{nM}$ for $48 \mathrm{~h}$, KIF5B-RET positive A549 cells hardly migrated in scratch assays, indicating the inhibiton of Dasatinib in migration. (E) Using Dasatinib with a concentration of $40 \mathrm{nM}$ could significantly inhibited migration and invasion in KIF5B-RET positive A549 cells, indicating that Src protein may be the downstream pathway of KIF5B-RET to mediate invasion and migration. ${ }^{*} P<0.05$. 
The biological functions of Apatinib in KIF5B-RET driven A549 cells were also investigated for the first time. In our study, we found Apatinib could inhibit the proliferation, migration and invasion in RET-rearranged LADC. But there was no obvious difference in inhibition efficiency of cell viability between KIF5B-RET transfected A549 cells and A549 cells. This might because the major effect of Apatinib in suppressing cell proliferation was performed through VEGF/ VEGFR signaling pathway in the transfected or parental A549 cell line, rather than through anti-RET pathway. The migration and invasion effects were significantly reduced in KIF5B-RET driven A549 cells, indicating Apatinib may be a promising anti-metastatic agent to reduce migration and invasion. As a RTK inhibitor targeting RET and c-Src, Apatinib suppressed autophosphorylation of RET and Src protein simultaneously in a concentration-dependent manner, showing that Apatinib exhibits potent inhibitory activity against RET fusion-positive cells by suppressing RET/Src pathway.
To date, more explorations are on-going in RET inhibitors to find the therapeutic potential as targeted drugs tailored for RET fusion-positive patients [23-26]. Although RET-inhibiting TKIs provide new therapeutic potential in RET-associated tumors, the adverse side effects that are associated with VEGFR or EGFR inhibition should not be ignored [27]. As the first generation of oral antiangiogenesis drug created in China, Apatinib has been known for its simplicity, compliance, and less side effects [28]. Presently, clinical trials of Apatinib in advanced NSCLC are on-going based on the similar mechanism by inhibiting vascular endothelial growth factor receptors [29]. Given our result that Apatinib inhibits cellular invasion and migration by fusion kinase KIF5B-RET, it may be possible of using Apatinib in tumors harboring RET gene fusions, especially those with metastatic diseases, which needs further animal studies or clinical trials to confirm.
A

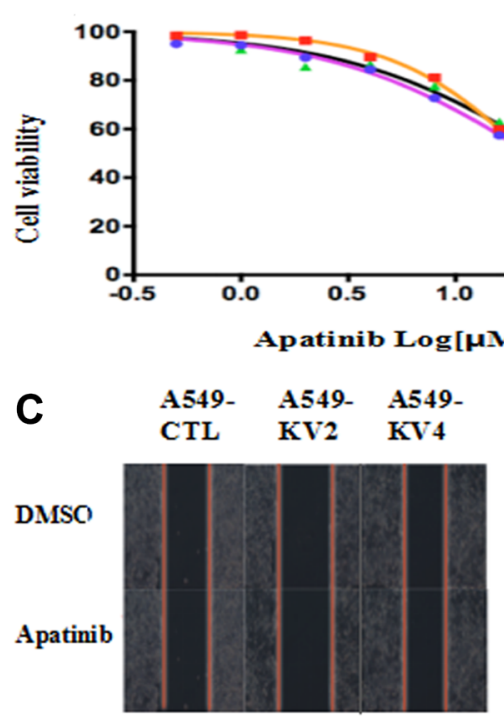

$\mathbf{0 h}$
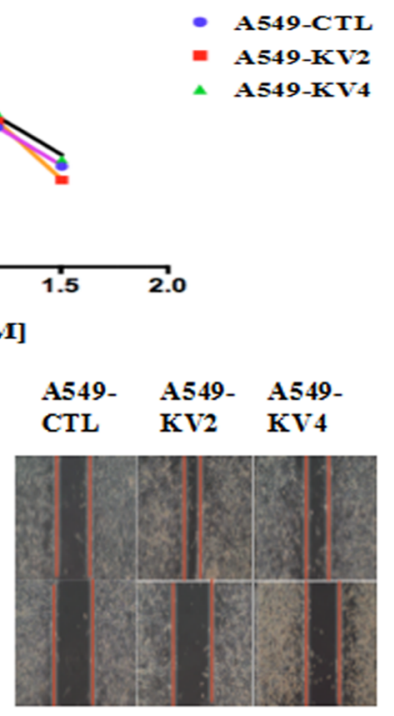

$48 h$

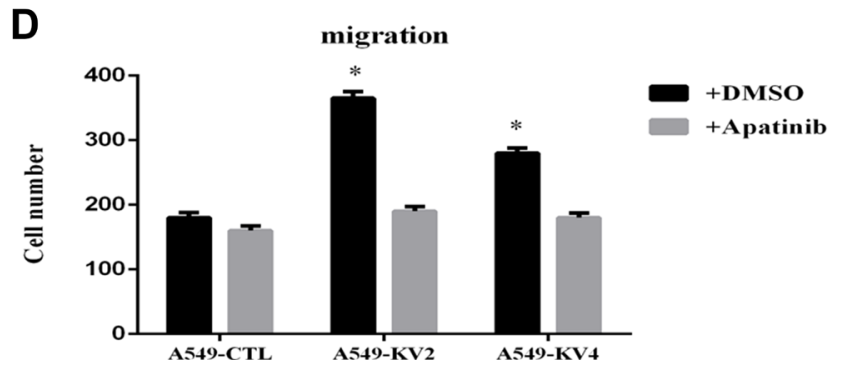

B
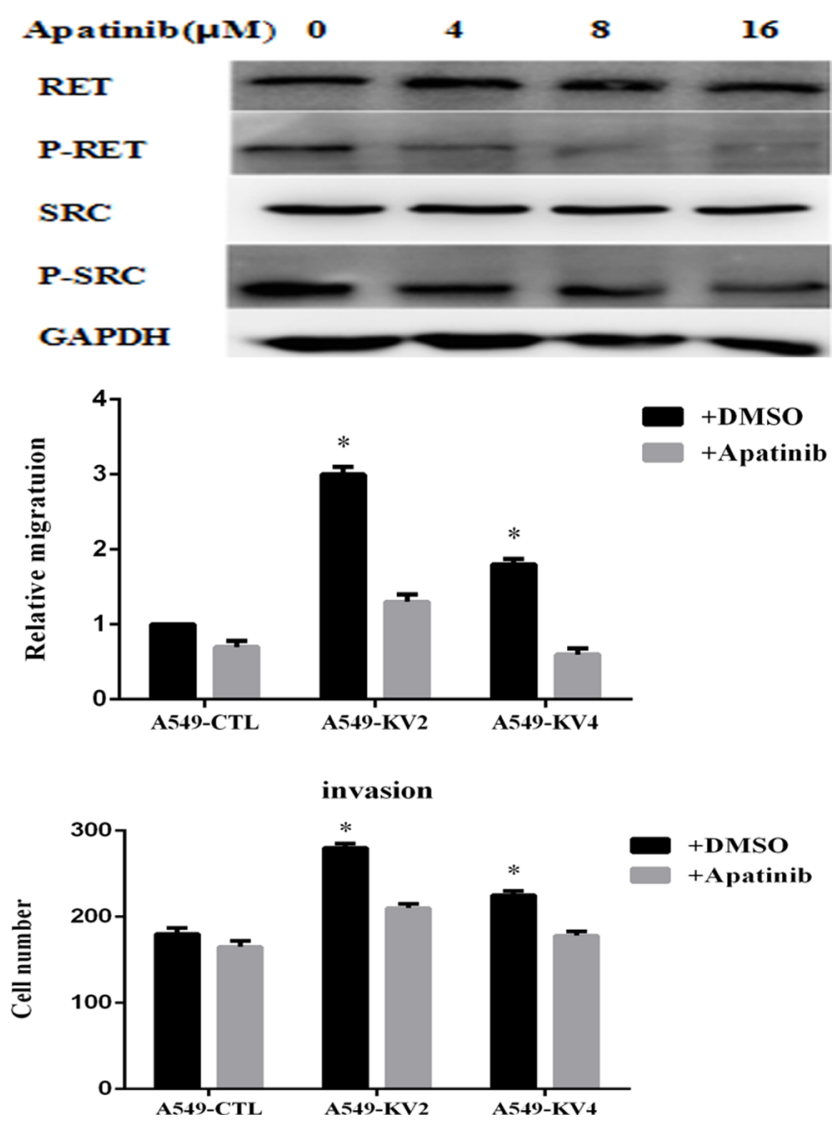

Figure 6: Apatinib inhibited cell proliferation, migration and invasion of KIF5B-RET transfected A549 cells. (A) KIF5B-RET transfected A549 cells and the negative control cells were treated with Apatinib at different concentration for 48 h, and found similar suppressed cell viability in a concentration-dependent manner but without obvious difference in inhibition efficiency. (B) Western Blot showed the variation of protein expression after treating cells with concentration-gradient Apatinib, and found a doseindependent reduction of the phosphorylation of RET gene fusion kinases and the p-RET was totally inhibited at the concentration of $8 \mu \mathrm{M}$, along with the inhibition of p-Src. (C) Treated with Apatinib at a concentration of $8 \mu \mathrm{M}$ for $48 \mathrm{~h}$, KIF5B-RET positive A549 cells hardly migrated in scratch assays, indicating the inhibiton of Apatinib in migration. (D) Using Apatinib with a concentration of $8 \mu \mathrm{M}$ could significantly inhibited migration and invasion in KIF5B-RET positive A549 cells. $* P<0.05$. 


\section{MATERIALS AND METHODS}

\section{Compounds and cell lines}

Human bronchial epithelial cell BEAS-2B, human lung cancer cells A549, and the mouse fibroblast cell NIH3T3 were obtained from the Cell Bank of Type Culture Collection of Chinese Academy of Sciences (Shanghai, China). Cells were cultured in DMEM or RPMI1640 supplemented with $10 \%$ fetal bovine serum (FBS) at $37^{\circ} \mathrm{C}$ in a humidified atmosphere with $5 \%$ $\mathrm{CO}_{2}$. Apatinib, PD98059 and Dasatinib were purchased from Selleck Chemical (Houston, TX, USA) at an initial concentration of $10 \mathrm{mM}, 20 \mathrm{mM}$ and $10 \mathrm{mM}$ (in $1 \mathrm{~mL}$ DMSO). Apatinib and PD98059 were directly used without dilution. Dasatinib was diluted to a final concentration of $50 \mu \mathrm{M}$. The final DMSO concentration in cell treatment was $0.04 \%-0.16 \%$.

\section{Lentivirus production and transduction}

Plasmids that express KIF5B-RET Variant2, KIF5BRET Variant4 were kindly gifted from Department of Thoracic Surgery, Fudan University Shanghai Cancer Center. Lentiviral vector system (Tronolab) was a four plasmid system, which was composed of pRsv-REV, pMDlg-pRRE, pMD2G, and target interference plasmid expressing green fluorescent protein (GFP). Cell line 293T, lentivirus packaging cell, was cultured in DMEM containing $10 \%$ FBS. E. coli strain DH5 $\alpha$ was used to amplify lentiviral vectors and help package vector plasmids. Stable transfected A549, Beas-2B and 3T3 cells were constructed by infection of KIF5B-RET-FLAG-expressing lentiviruses.

\section{Cell proliferation assays}

BEAS-2B and A549 cells were seeded at a density of 2000 cells and 500 per well in $96-w e l l$ plates. The cells were transfected with KIF5B-RET Variant2 or KIF5BRET Variant4. Cell proliferation was analyzed using Cell Counting Kit 8 (Dojindo, Kumamoto, Japan) according to the manufacturer's protocol.

\section{Colony formation assays}

A549 cells were seeded into 6-well plates at a concentration of 5000 cells per well. Cells were cultured for 10 days. At the end of incubation, cells were fixed with methanol for $10 \mathrm{~min}$ and stained with crystal violet for $10 \sim 15 \mathrm{~min}$.

\section{Scratch assays}

Cells were seeded in a 6-well plate, and a "wounding" line was scratched in the center of the cell monolayers with a sterile $200-\mu \mathrm{L}$ pipette tip. The debris was removed by washing at least twice with PBS. Serum
- free medium were added.The width of the wound was measured under a microscope at 0 and 48 hours after the scratch to assess the migration ability of the cells.

\section{Cell migration and invasion assays}

Migration and invasion experiments in vitro were carried out in the chamber of $8-\mu \mathrm{m}$ transwell inserts (BD Falcon ${ }^{\mathrm{TM}}$; Becton Dickinson, Franklin Lakes, NJ, USA) with or without Matrigel (BD Falcon ${ }^{\mathrm{TM}}$ ). An amount of $10^{5}$ A549 cells were incubated in serum-free medium at the top chamber of each well insert, and serum-containing medium were added to the lower chamber. Cells that migrated were fixed in $10 \%$ formalin and stained with $1 \%$ crystal violet after 24 hours of incubation at $37^{\circ} \mathrm{C}$, and were counted under a light microscope at a magnification of $\times 200$.

\section{Western blot}

Total protein lysates were obtained from cultured cells using radio-immunoprecipitation assay buffer supplemented with complete protease inhibitor cocktail (Roche, Basel, Switzerland). Protein concentrations were determined using the BCA protein assay kit (Biyotime, Shanghai, China). Cell extracts were subjected to sodium dodecyl sulfatepolyacrylamide gel electrophoresis and were transferred into polyvinylidene fluoride (PVDF). The membrane was then blocked with $5 \%$ skim milk in TBST for $2 \mathrm{~h}$ at room temperature and probed with the primary antibodies overnight at $4^{\circ} \mathrm{C}$. After washing with TBST, the membrane was incubated with horseradish peroxidase (HRP)-conjugated secondary antibody for $1 \mathrm{~h}$ at room temperature, washed three times with TBST, and detected by enhanced chemiluminescence reagent (Pierce, Rockford, IL, USA).Antibodies against RET, p-RET, ERK, p-ERK, Src, p-Src, GAPDH, $\beta$-actin were purchased from Cell Signaling Technology (Cambridge, MA, USA).

\section{Xenograft tumor model and hematoxylin-eosin staining}

$5 \times 10^{6}$ NIH3T3 cells carrying KIF5B-RET Variant 2, KIF5B-RET Variant 4 and the control NIH3T3 cells were injected subcutaneously to 6-week-old female nude mice. The tumors were measured every week until 1 month later. The xenograft tumor samples were fixed in $10 \%$ neutral-buffered formalin for $24 \mathrm{~h}$ and embedded in paraffin. Sections were reacted with hemalum for nuclear staining, and then counterstained with eosin for staining of other eosinophilic structures.

\section{Statistical analysis}

All experiments were repeated at least thrice. Statistical analysis was performed using Statistical Package for the Social Sciences (SPSS) software version 22.0 for 
Windows (SPSS Inc., Chicago, USA). Differences between groups were calculated by using the Student's $t$-test. The statistical significance was determined at $P$-value $<0.05$. Graphs were created with GraphPad Prism 5.

\section{Abbreviations}

NSCLC-Non-Small Cell Lung Cancer; RETRearranged during Transfection; KIF5B-Kinesin Family 5B; LADC-Lung Adenocarcinomas; TKIs-Tyrosine Kinase Inhibitors; RTK-Receptor Tyrosine Kinase; VEGFR2-Vascular Endothelial Growth Factor Receptor-2; PDGFR- $\beta$-Platelet-Derived Growth Factor- $\beta$; c-Src-vSrc Sarcoma Viral Oncogene Homolog; c-Kit-Stem Cell Factor Receptor; FBS-Fetal Bovine Serum; GFP-Green Fluorescent Protein; PVDF-Polyvinylidene Fluoride; HRPHorseradish Peroxidase; SPSS-Statistical Package for the Social Sciences; MMPs-Matrix Metalloproteninases; FAK-Focal Adhesion Kinase.

\section{ACKNOWLEDGMENTS}

This work is supported by Shanghai Science and Technology Committee (Grant No. 124119a6200).

\section{CONFLICTS OF INTEREST}

The authors declare no conflicts of interest.

\section{Authors' contributions}

Conceived and designed the experiments: Jianhua Chang. Performed the experiments and Analyzed the data: Shanshan Wang, Weiwei Xie, Rongliang Zheng. Technical and editorial support: Yu Gan. Wrote the paper: Chen Lin.

\section{REFERENCES}

1. Gainor JF, Shaw AT. Novel targets in non-small cell lung cancer: ROS1 and RET fusions. Oncologist. 2013; 18:865-75.

2. Pan Q, Wang Y, Chen J, Xu G, Chen B, Pan J, Huang K. Investigation of the epidermal growth factor receptor mutation rate in non-small cell lung cancer patients and the analysis of associated risk factors using logistic regression. Oncology Letters. 2014; 8:813-8.

3. Awad MM, Shaw AT. ALK inhibitors in non-small cell lung cancer: crizotinib and beyond. Clin Adv Hematol Oncol. 2014; 12:429-39.

4. Girard N. Other signalization targets. Targ Oncol. 2013; $8: 3-14$.

5. Shtivelman E, Hensing $T$, Simon GR, Dennis PA, Otterson GA, Bueno R, Salgia R. Molecular pathways and therapeutic targets in lung cancer. Oncotarget. 2014. 30; 5:1392-433. doi:10.18632/oncotarget.1891.
6. Kohno T, Ichikawa H, Totoki Y, Yasuda K, Hiramoto M, Nammo T, Sakamoto H, Tsuta K, Furuta K, Shimada Y, Iwakawa R, Ogiwara H, Oike T, et al. KIF5B-RET fusions in lung adenocarcinoma. Nat Med. 2012; 18:375-7.

7. Takeuchi K, Soda M, Togashi Y, Suzuki R, Sakata S, Hatano S, Asaka R, Hamanaka W, Ninomiya H, Uehara H, Lim Choi Y, Satoh Y, Okumura S, et al. RET, ROS1 and ALK fusions in lung cancer. Nat Med. 2012; 18:378-81.

8. Lipson D, Capelletti M, Yelensky R, Otto G, Parker A, Jarosz M, Curran JA, Balasubramanian S, Bloom T, Brennan KW, Donahue A, Downing SR, Frampton GM, et al. Identification of new ALK and RET gene fusions from colorectal and lung cancer biopsies. Nat Med. 2012; 18:382-4.

9. Ju YS, Lee WC, Shin JY, Lee S, Bleazard T, Won JK, Kim YT, Kim JI, Kang JH, Seo JS. A transforming KIF5B and RET gene fusion in lung adenocarcinoma revealed from whole- genome and transcriptome sequencing. Genome Res. 2012; 22:436-445.

10. Phay JE, Shah MH. Targeting RET Receptor Tyrosine Kinase Activation in Cancer. Clin Cancer Res. 2010; 16:5936-41.

11. Pützer BM, Drosten M. The RET proto-oncogene: a potential target for molecular cancer therapy. Trends Mol Med. 2004; 10:351-7.

12. Song M. Progress in Discovery of KIF5B-RET Kinase Inhibitors for the Treatment of Non-Small-Cell Lung Cancer. J Med Chem. 2015; 58:3672-81.

13. Tong XZ, Wang F, Liang S, Zhang X, He JH, Chen XG, Liang YJ, Mi YJ, To KK, Fu LW. Apatinib (YN968D1) enhances the efficacy of conventional chemotherapeutical drugs in side population cells and ABCB1-overexpressing leukemia cells. Biochem Pharmacol. 2012; 83:586-97.

14. Ruixuan Geng, Jin Li. Apatinib for the treatment of gastric cancer. Expert Opin Pharmacother. 2015; 16:117-22.

15. Hicklin DJ, Ellis LM. Role of the vascular endothelial growth factor pathway in tumor growth and angiogenesis. J Clin Oncol. 2005; 23:1011-27.

16. Li J, Qin S, Xu J, Xiong J, Wu C, Bai Y, Liu W, Tong J, Liu Y, Xu R, Wang Z, Wang Q, Ouyang X, et al. J Clin Oncol. 2016; 34:1448-54.

17. Tsai TH, Wu SG, Hsieh MS, Yu CJ, Yang JC, Shih JY. Clinical and prognostic implications of RET rearrangements in metastatic lung adenocarcinoma patients with malignant pleural effusion. Lung Cancer. 2015; 88:208-14.

18. Wang R, Hu H, Pan Y, Li Y, Ye T, Li C, Luo X, Wang L, Li H, Zhang Y, Li F, Lu Y, Lu Q, et al. RET fusions define a unique molec-ular and clinicopathologic subtype of nonsmall-cell lung cancer. J Clin Oncol. 2012; 30:4352-9.

19. Tsuta K, Kohno T, Yoshida A, Shimada Y, Asamura H, Furuta K, Kushima R. RET-rearranged non-small-cell lung carcinoma: a clinicopathological and molecularanalysis. $\mathrm{Br}$ J Cancer. 2014; 110:1571-8.

20. Qian Y, Chai S, Liang Z, Wang Y, Zhou Y, Xu X, Zhang C, Zhang M, Si J, Huang F, Huang Z, Hong W, Wang K. 
KIF5B-RET fusion kinase promotes cell growth by multilevel activation of STAT3 in lung cancer. Mol Cancer. 2014; $13: 176$.

21. Liu W, Kovacevic Z, Peng Z, Jin R, Wang P, Yue F, Zheng M, Huang ML, Jansson PJ, Richardson V, Kalinowski DS, Lane DJ, Merlot AM, et, al. The molecular effect of metastasis suppressors on Src signaling and tumorigenesis: new therapeutic targets. Oncotarget. 2015; 6:35522-41. doi: 10.18632/oncotarget.1891.

22. Yeatman TJ. A renaissance for SRC. Nat Rev Cancer. 2004; 4:470-80.

23. Borrello MG , Ardini E, Locati LD, Greco A, Licitra L, Pierotti MA. RET inhibition: implications in cancer therapy. Expert Opin. Ther. Targets. 2013; 17:403-19.

24. Mukhopadhyay S, Pennell NA, Ali SM, Ross JS, Ma PC, Velcheti V. RET-Rearranged Lung Adenocarcinomas with Lymphangitic Spread, Psammoma Bodies, and Clinical Responses to Cabozantinib. J Thorac Oncol. 2014; 9:1714-9.
25. Gautschi O, Zander T, Keller FA, Strobel K, Hirschmann A, Aebi S, Diebold J. A patient with lung adenocarcinoma and RET fusion treated with vandetanib. J Thorac Oncol. 2013; 8:e43-4.

26. Song M, Kim SH, Yoon SK. Cabozantinib for the treatment of non-small cell lung cancer with KIF5B-RET fusion. An example of swiftrepositioning. Arch Pharm Res. 2015; 38:2120-3.

27. Mologni L. Development of RET kinase inhibitors for targeted cancer therapy. Curr Med Chem. 2011; 18:162-75.

28. Liu L, Yu H, Huang L, Shao F, Bai J, Lou D, Chen F. Progression-free survival as a surrogate endpoint for overall survival in patients with third-line or later-line chemotherapy for advanced gastric cancer. Onco Targets Ther. 2015; 8:921-928.

29. Zhang H. Apatinib for molecular targeted therapy in tumor. Drug Des Devel Ther. 2015; 13:6075-81. 